\title{
Regulation of aspartate-derived amino acid homeostasis in potato plants (Solanum tuberosum L.) by expression of $E$. coli homoserine kinase
}

\author{
J. Rinder ${ }^{1}$, A. P. Casazza ${ }^{2}$, R. Hoefgen ${ }^{1}$, and H. Hesse ${ }^{1}$ \\ ${ }^{1}$ Department of Molecular Physiology, Max-Planck-Institut für Molekulare Pflanzenphysiologie, Golm, Germany \\ ${ }^{2}$ Department of Biology, Section of Plant Physiology and Biochemistry, University of Milan, Milan, Italy
}

Received November 10, 2006

Accepted February 6, 2007

Published online July 12, 2007; (C) Springer-Verlag 2007

\begin{abstract}
Summary. The availability of the carbon backbone $O$-phosphohomoserine (OPHS) is critical to methionine (met) and threonine (thr) synthesis. OPHS derives from homoserine and is formed by homoserine kinase (HSK). To clarify the function of HSK in cellular metabolism, the E. coli HSK ortholog $t h r B$ was expressed in potato plants targeting the EcHSK protein to chloroplasts and to the cytosol. Both approaches resulted in up to 11 times increased total HSK enzyme activity. Transgenic plants exhibited reduced homoserine levels while met and thr did not accumulate significantly. However, the precursor cysteine and upstream intermediates of met such as cystathionine and homocysteine did indicating an accelerated carbon flow towards the end products. Coincidently, plants with elevated cytosolic levels of EcHSK exhibited a reduction in transcript levels of the endogenous $H S K$, as well as of threonine synthase $(T S)$, cystathionine $\beta$-lyase $(C b L)$, and met synthase $(M S)$. In all plants, cystathionine $\gamma$-synthase $(\mathrm{CgS})$ expression remained relatively unchanged from wild type levels, while $S$-adenosylmethionine synthetase (SAMS) expression increased. Feeding studies with externally supplied homoserine fostered the synthesis of met and thr but the regulation of synthesis of both amino acids retained the wild type regulation pattern. The results indicate that excess of plastidial localised HSK activity does not influence the de novo synthesis of met and thr. However, expression of HSK in the cytosol resulted in the down-regulation of gene expression of pathway genes probably mediated via OPHS. We integrated these data in a novel working model describing the regulatory mechanism of met and thr homeostasis.
\end{abstract}

Keywords: Aspartate family - Homoserine kinase - Methionine Threonine - Potato

\section{Introduction}

The four essential amino acids of the aspartate family are methionine (met), lysine (lys), threonine (thr), and isoleucine (ile). In plants, this pathway branches at two points: 3-aspartic acid (towards lys) and $O$-phosphohomoserine (OPHS; towards met, thr, and ile). In microorganisms, OPHS is used exclusively for thr synthesis. The branch point is homoserine in yeast and succinyl-homoserine in bacteria (Hesse et al., 2004).

The synthesis of aspartate-derived amino acids is subject to complex regulation. The key to pathway control is feedback inhibition of aspartate kinase by lys and/or thr, or by lys in concert with $S$-adenosylmethionine (SAM). Further, each branch is individually regulated. Homoserine kinase (HSK) catalyses the formation of OPHS, which then serves as substrate for cystathionine gamma-synthase $(\mathrm{CgS})$ and threonine synthase (TS). CgS and TS are regulated by SAM at the transcript (CgS in Arabidopsis; Chiba et al., 1999) or protein level (TS in Arabidopsis and potato; Curien et al., 1996, 1998; Zeh et al., 2001), depending on the plant species (for review see Hesse and Hoefgen, 2003).

Reverse and forward approaches indicate that amino acid pools likely fill in a controlled order. The lys pool is filled before the met pool, and finally thr/ile (Galili, 1995; Hesse and Hoefgen, 2003; Hesse et al., 2004). Carbon provided by asp via OPHS is used preferentially to form met. When met levels are high, SAM levels modulate the affinity of TS for OPHS to levels 250-500 fold lower than the $K_{\mathrm{m}}$ value of $\mathrm{CgS}$ for the common substrate (Curien et al., 1998). Thus, accumulation of SAM redirects the carbon backbone towards thr synthesis. Earlier studies have shown that elevated levels of the carbon backbone (e.g. over-expression of aspartate kinase) increase the synthesis of lys and thr (Galili, 1995; Galili et al., 2005). This suggests that carbon flux limits the synthesis of met, which itself implies that met levels could be increased if OPHS were available in sufficient amounts. Cys, as the donor of reduced sulfur, seems to have only 
a minor effect on met synthesis (Nikiforova et al., 2002; Riemenschneider et al., 2005).

HSK is a low abundance enzyme and, thus, is challenging to investigate in much detail, although Riesmeier et al. (1993) were able to purify and characterise native HSK from wheat. The regulation of the enzyme is variable. In wheat, it is not inhibited by physiological concentrations of thr, met, val, ile, or SAM (Riesmeier et al., 1993). Older studies, however, show that HSK from pea and radish is allosterically inhibited by these amino acids (Thoen et al., 1978; Baum et al., 1983). Greenberg et al. (1988) isolated soybean cell lines with HSK activity repressed due to met accumulation.

HSK localised to pea chloroplasts appears to contain both soluble and membrane-associated activities (Muhitch and Wilson, 1983; Wallsgrove et al., 1983). Further support for chloroplastic localisation was provided by the isolation of HSK in Arabidopsis: a single gene locus was identified and the predicted enzyme has a chloroplast targeting sequence and a mature mass of $33 \mathrm{kDa}$. The recombinant protein was characterised biochemically and found to be dependent on $\mathrm{Mg}^{2+}$ and $\mathrm{K}^{+}$ions (Lee and Leustek, 1999).

A portion of the homoserine pool is exported from chloroplasts and translocated to other plant organs. In phloem exudates from pea plants, homoserine is one of the major amino acids transported to seeds, along with asn and gln (Rochat and Boutin, 1991). Furthermore, studies by Aubert et al. (1998) for sycamore (Acer pseudoplatanus) and the monocotyledonous weed Echinochloa colonum show that OPHS is present in the cytosol. These authors used a ${ }^{31} \mathrm{P}-\mathrm{NMR}$ technique that made it possible to discriminate between OPHS in the cytosol and in the chloroplasts based on a shift in the OPHS resonance spectrum caused by different $\mathrm{pH}$-values. Homoserine (or its derivatives) may also function in planta as a host signal for the expression of pathogenesis genes such as pelD in Nectria haematococca (Yang et al., 2005).

To gain knowledge of the role of HSK and its possible effect on OPHS levels, especially in the context of cellular compartment, we applied a transgenic approach in potato. We targeted the expression of the E. coli HSK ortholog to either chloroplasts or the cytosol. We identified several plants with increased total HSK activity. Under normal growth conditions, these plants have generally phenotypes like wild type, regardless of tentatively increases in met and thr levels obtained for both subcellular targeted EcHSK constructs. These data suggest that OPHS is able to cross the chloroplast membrane as shown by higher internal met and thr levels and that increases in the OPHS pool promote the synthesis of met and thr as shown by homoserine feeding experiments. Furthermore, the data suggest that OPHS or homoserine exported to the cytosol might have a regulatory function directly correlating to met and thr homeostasis.

\section{Materials and methods}

Construction of a chimeric EcHSK gene and potato transformation

DNA manipulations were carried out as described by Sambrook et al. (1989). A DNA fragment encoding the mature protein of HSK (thrB) from E. coli $\mathrm{K} 12$ (M15745) was amplified by polymerase chain reaction generating a $\mathrm{NcoI}$ site at its $5^{\prime}$-end and a $\mathrm{XbaI}$ site at its $3^{\prime}$-end (oligonucleotides for $H S K$ : $E c H S K$-N: $5^{\prime}-E c H S K-\mathrm{N}$ : $5^{\prime}$-GAG ACC ATG GTT AAA GTT TAT GCC CC; $E c H S K$-C: $5^{\prime}$-AGA GAT CTA GAA TAG TTT TCC AGT ACT CG; TIB MOLBIOL, Berlin, Germany). Amplified fragments encoding for the mature protein were ligated into a pUC18 derivative according to Harms et al. (2000) containing the $r b c S$ signal sequence from Arabidopsis thaliana in order to provide a targeting sequence to chloroplasts. The fused gene product was inserted as an Asp718/XbaI fragment into an Asp718/XbaI pre-digested binary vector (Höfgen and Willmitzer, 1990) under the control of the 35S-CaMV promoter to obtain transgenic plants expressing EcHSK in chloroplasts.

For cytosolic targeting of EcHSK, the DNA fragment cloned in pUC18 was cleaved with $\mathrm{NcoI}$ and than blunted in a fill-in reaction by the Klenow fragment. After additional digestion with $X b a \mathrm{I}$, the HSK fragment was excised and cloned into the pre-digested $S m a \mathrm{I} / \mathrm{X} b a \mathrm{I}$ pBinAR vector.

Transformation of the potato cultivar Désirée was performed via Agrobacterium tumefaciens according to Rocha-Sosa et al. (1989) using the strain C58C1/pGV2260 (Deblaere et al., 1985). Transgenic plants were selected on medium containing kanamycin $\left(10 \mathrm{mgl}^{-1}\right)$ according to Murashige and Skoog (1962).

\section{Plant cultivation}

Transgenic EcHSK-expressing plants were propagated in tissue culture along with potato wild type plants and transferred into soil after 2 weeks of cultivation. The rooted shoots were planted in small pots and grown in the phytotron with a light regime of $200-250 \mu \mathrm{mol} \mathrm{s}^{-1} \mathrm{~m}^{-1}(16 \mathrm{~h} / 8 \mathrm{~h})$ under a hood to retain high air humidity. After 2 weeks, plants were transferred into pots with a diameter of $20 \mathrm{~cm}$ and cultivated in a greenhouse providing nearly natural light conditions with an approximately 16-h-light/8-hdark period plus natural sunlight. Light intensity and temperature were dependent on environmental conditions, but light did not fall below 250$300 \mu \mathrm{mol}$ photons $\mathrm{m}^{-2} \mathrm{~s}^{-1}$, and temperature did not sink below $18^{\circ} \mathrm{C}$. Leaf material was harvested from greenhouse-grown plants after approximately 8 weeks of cultivation, before the onset of flowering. Leaf discs were excised from tissues of similar developmental stage. Transition to the reproductive stage could usually be observed only in plants older than 10 weeks. All plant material was sampled in the morning and immediately frozen in liquid nitrogen before storage at $-80^{\circ} \mathrm{C}$.

\section{RNA extraction, cDNA synthesis and semi-quantitative PCR}

Total RNA was extracted essentially as described by Logemann et al. (1987). RNA samples $(50 \mu \mathrm{g})$ were separated on $1.2 \%$ denaturing agaroseformaldehyde gels. Equal loading was controlled by staining the gels with ethidium bromide. Gels were blotted to nylon membranes Biodyne B Transfer membrane (Pall, Portsmouth, England), hybridised with radioactive ${ }^{32} \mathrm{P}$-dCTP-labelled EcHSK DNA fragment. Washing was performed under stringent conditions $\left(2 \times \mathrm{SSC}\right.$ for $30 \mathrm{~min}$ at $65^{\circ} \mathrm{C}$ and $0.1 \times \mathrm{SSC}$, 
$0.1 \%$ SDS at $65^{\circ} \mathrm{C}$ for $15 \mathrm{~min}$ ) and membranes were exposed to X-ray film (Kodak X-Omat AR film) at $-80^{\circ} \mathrm{C}$.

In order to analyse expression of aspartate pathway related genes in the EcHSK over-expressing plants and wild type control plants, RNA was extracted for semi-quantitative PCR. A two-step semi-quantitative PCR method was used to measure gene expression in leaf tissue samples at the time of harvest. Oligo-(dT) ${ }_{15}$ (Promega, Germany) was used as primer in the first step of cDNA synthesis. Total RNA $(100 \mu \mathrm{g})$ was combined with $1 \mu \mathrm{g}$ oligo-dT, $200 \mu \mathrm{M}$ dNTPs and $\mathrm{H}_{2} \mathrm{O}$ and preheated at $65^{\circ} \mathrm{C}$ for 2 min to denature secondary structures. The mixture was cooled rapidly and then $10 \mu 15 \times$ RT buffer, $10 \mathrm{mM}$ DTT and $200 \mathrm{U}_{\text {SuperScript }}{ }^{\mathrm{TM}}$ III RT (Invitrogen, Germany) was added for a total volume of $50 \mu$. The RT mix was incubated at $37^{\circ} \mathrm{C}$ for $90 \mathrm{~min}$ then stopped by heating at $95^{\circ} \mathrm{C}$ for $5 \mathrm{~min}$. The cDNA stock was stored at $-20^{\circ} \mathrm{C}$. The yield of cDNA was measured according to the PCR signal generated from the internal standard housekeeping gene actin amplified from 20 to 35 cycles starting with $2 \mu \mathrm{l}$ of the cDNA solution. The volume of each cDNA pool was adjusted to give the same exponential phase PCR signal strength for actin after 20 cycles.

Relative PCR was performed to measure gene expression of pathwayrelevant genes. DNA fragments were amplified by polymerase chain reaction generating fragments of about 500 bases (oligonucleotides for HSK: $S t H S K$-N $5^{\prime}$ GCA ATA ACC TAC CAA TCT CCC, $S t H S K$-C $5^{\prime}$ CCG ATT CCA ACC CAG CAA GC; TS: StTS-N 5' CCT CTT TCA TCT CTC CTG GTC TTC CTC, StTS-C 5' CTT AGA CCA AAC ACC GGA CCC ATA AGG CC; CgS: $S t C g S-N 5^{\prime}$ CAA GTT GTT GCG GCT TCC TGG TCT AA, $S t C g S-C 5^{\prime}$ GGC ACA CAT TCC AGA TGC CAC TAT CAA GGT; CbL: $S t C b L-\mathrm{N} 5^{\prime}$ GCT CTT TGC CTA CCA ACA CAA CCA CCC AGG, $S t C b L-C 5^{\prime}$ CCC CGG CAA CAA TCT CCT CAC CAG CTT TAA; MS: $S t M S$-N $5^{\prime}$ GGG CCC AAA GAG AGA GCT GAA ATT CGC TC, StMSN 5' GGG AAA GAT TTC TCA ACA CCC TTA GCA GGC; SAMS: StSAMS-N 5' GCC TAG AGC AGG ACC CTG AAA GCA AGG TCG, StSAMS-C $5^{\prime}$ TCT TGC CAT CGG GCC TGA GCC ATG GGC AGG; Stactin-N $5^{\prime}$ CCC TAG CAT TGT GGG ACG TCC TCG TCA CAC, Stactin-C $3^{\prime}$ CCG CTC GGC TGT GGT GGT GAA AGA GTA GCC; TIB MOLBIOL, Berlin, Germany).

Polymerase chain reactions were performed on a Biometra PCR machine (Germany) using $\sim 100 \mathrm{ng}$ of cDNA, $0.2 \mu \mathrm{M}$ specific primers, $1 \mathrm{mM}$ $\mathrm{MgCl}_{2}, 2 \mathrm{mM}$ dNTPs, $1 \times$ PCR buffer and $5 \mathrm{U}$ Taq Polymerase (Gibco BRL, Germany) in a $100 \mu \mathrm{l}$ volume. After $20,25,30,35$, and 40 cycles, $5 \mu \mathrm{l}$ samples were taken for electrophoresis. The PCR samples were electrophoresed on $1 \%$ agarose containing ethidium bromide for staining and photographed on top of a $280 \mathrm{~nm}$ UV light box. The gel images were digitally captured with a CCD camera.

\section{Determination of enzyme activity}

Total HSK activity was measured as described by Lee and Leustek (1999). Leaf tissues $(100 \mathrm{mg})$ from source leaves of 5 replica 8-week-old plants, 1 sample each, were collected and immediately frozen in liquid nitrogen. The samples were then kept at $-80^{\circ} \mathrm{C}$ until the assay was performed. The extraction buffer consisted of $50 \mathrm{mM} \mathrm{KH_{2 }} \mathrm{HPO}_{4}, \mathrm{pH} 7.5 ; 0.5 \mathrm{mM}$ EDTA; 5 mM DTT; $15 \%$ Glycerol; $0.1 \%$ Triton X-100.

Assays were initiated by adding the protein extract to the reaction buffer containing $50 \mathrm{mM}$ MOPS/ $\mathrm{HCl}, \mathrm{pH} 8.5 ; 100 \mathrm{mM} \mathrm{KCl} ; 1.5 \mathrm{mM} \mathrm{MgCl}_{2}$; $2.5 \mathrm{mM}$ ATP; $18 \mathrm{mM}$ homoserine. After incubating the mixture for 0,3 , and $6 \mathrm{~min}$ at $25^{\circ} \mathrm{C}$, aliquots were taken and the reaction was stopped by adding 20\% TCA. OPHS formation was analysed by high-performance liquid chromatography (HPLC) after derivatisation with $o$-phthaldialdehyde (OPA). The column was calibrated with OPHS (ChiroBlock GmbH, Wolfen, Germany). In alkaline solutions OPHS is not stable. Thus, the derivatisation protocol according to Kreft et al. (2003) was modified. Instead of adding the borate buffer directly to the extracted amino acids, OPA and borate buffer were added and mixed directly before injection to the HPLC system. The given reaction time was sufficient to determine OPHS
Extraction and analysis of soluble amino acids

Leaf tissues (about $100 \mathrm{mg}$ per plant) were ground to a fine powder in liquid nitrogen in a bead mill and extracted three times for $20 \mathrm{~min}$ at $80^{\circ} \mathrm{C}$ : once with $400 \mu \mathrm{l}$ of $80 \%$ (v/v) aqueous ethanol (buffered with $2.5 \mathrm{mM}$ HEPES-KOH, $\mathrm{pH} 7.5$ ), once with $400 \mathrm{ml}$ of $50 \%(\mathrm{v} / \mathrm{v})$ aqueous ethanol (buffered as before), and once with $200 \mu 1$ of $80 \%$ (v/v) aqueous ethanol. Between the extraction steps, the samples were centrifuged for $10 \mathrm{~min}$ at $13,000 \times g$, and the supernatants collected. The combined etha$\mathrm{nol} /$ water extracts were stored at $-20^{\circ} \mathrm{C}$ or directly subjected to RPHPLC using an ODS column (Hypersil C18; $150-\mathrm{mm} \times 4.6-\mathrm{mm}$ i.d.; $3 \mu \mathrm{m}$; Knauer GmbH, Berlin) connected to an HPLC system (Dionex, Idstein, Germany). Amino acids were measured by precolumn derivatization with OPA in combination with fluorescence detection (Lindroth and Mopper, 1979) as described by Kreft et al. (2003). Peak areas were integrated using Chromeleon 6.30 software (Dionex) and subjected to quantification by means of calibration curves made from standard mixtures.

\section{Feeding experiments with detached potato petioles}

Feeding experiments were carried out with potato petioles cut directly from 8-week-old greenhouse grown plants. To avoid xylem embolism, the potato petioles were cut again under a buffer solution (10 mM MES, $1 \mathrm{mM}$ EDTA, pH 6.5) and then immediately transferred to fresh buffer containing 0.1 and $1 \mathrm{mM}$ dissolved homoserine, respectively. Detached potato petioles were incubated in buffer without homoserine as a control. After $24 \mathrm{~h}$ incubation in constant light, leaf disks were taken from the petioles and analysed by HPLC as described above.

\section{Repetitions of experiments and statistical analysis of data}

All experiments were repeated two to three times with independent sets of greenhouse-grown plants. Student's $t$-tests were performed using the algorithm incorporated into Microsoft Excel 97. The term 'significant' is used only when a change in question has been confirmed to be statistically significant $(P<0.05)$ with the $t$-test.

\section{Results}

\section{Engineering and screening of plants with increased HSK enzyme activity}

In order to increase the activity of HSK, potato plants were transformed with the vector pBinAR harbouring the $E$. coli thrB gene encoding HSK. EcHSK was targeted either to the cytosol or to chloroplasts by fusion to an appropriate transit peptide (Harms et al., 2000) under the control of the CaMV $35 \mathrm{~S}$ promoter. Eighty independent transgenic plants were generated per construct. During their growth phase, plants were evaluated for macroscopic phenotypic alterations. Macroscopically, the transgenic plants were indistinguishable from wild type plants (data not shown). Transgenic lines were selected with respect to increased EcHSK mRNA levels. For all investigated transgenic lines, a substantial increase in their EcHSK transcript levels over wild type was determined (Fig. 1A). Differences in RNA size resulted from the addition of the transit peptide to the chloroplasttargeted construct. 


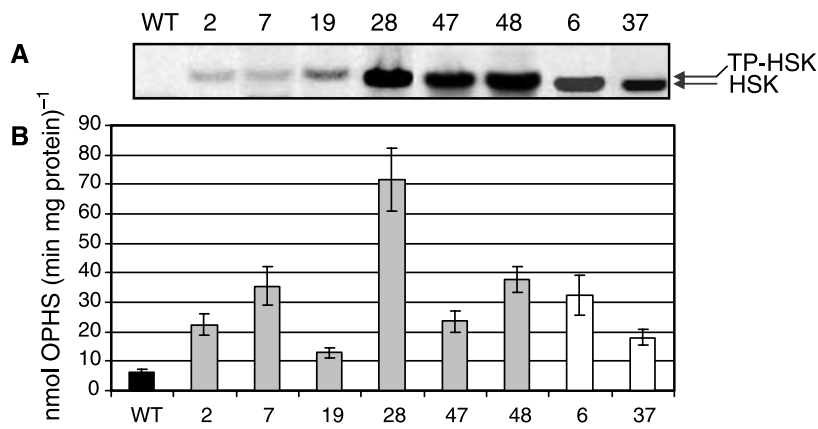

Fig. 1. Analysis of EcHSK mRNA levels and total HSK activity in control plants (WT) and independent EcHSK over-expressing lines (plastidial targeting: 2, 7, 19, 28, 47, 48, cytosolic targeting: 6, 37). A Total leaf RNA ( $50 \mu \mathrm{g}$ per lane) was separated on a $1.2 \%$ formaldehyde agarose gel and blotted onto a nylon membrane. mRNA was detected using a specific ${ }^{32} \mathrm{P}$-labelled EcHSK including the transpeptide. The transcript size was approximately $0.9 \mathrm{~kb}$. B Total extracts of soluble proteins were prepared from wild type (black bar) and transgenic plants (plastidial localisation: grey bars; cytosolic localisation: white bars). Total HSK activity was determined by measuring the formation of OPHS by HPLC. Activities are given as $\mathrm{nmol}$ ( $\mathrm{min} \times \mathrm{mg}$ protein)

Plants exhibiting higher EcHSK RNA levels were also tested for total HSK activity, and showed elevated levels in all cases (Fig. 1B). HSK activity was determined in crude protein extracts of controls and of transgenic lines $2,7,19,28,47$, and 48 expressing the chloroplast-targeted EcHSK construct, and lines 6 and 37 expressing the cytosol-targeted construct. Wild type crude extracts had HSK activities of about $6.3 \mathrm{nmol}(\mathrm{mg}$ protein/min). In lines expressing the chloroplast-targeted construct, activities increased to $22.5 \mathrm{nmol}$ (mg protein/min; line 2), $35.4 \mathrm{nmol}$ (mg protein/min; line 7), $12.9 \mathrm{nmol}$ (mg protein/min; line 19), $71.7 \mathrm{nmol}$ (mg protein/min; line 28), $23.6 \mathrm{nmol}(\mathrm{mg}$ protein $/ \mathrm{min}$; line 47 ), and $37.9 \mathrm{nmol}$ (mg protein/ min; line 48; Fig. 2B). In the cytosol-targeted lines, levels reached $32.5 \mathrm{nmol}$ (mg protein $/ \mathrm{min}$; line 6 ) and $18.0 \mathrm{nmol}$ (mg protein/min; line 37). The success of chloroplast targeting was tested according to Harms et al. (2000) (data not shown). Subcellular fractionation of leaves from transgenic and wild type plants and successive enzyme measurements revealed that most of the HSK activity was located either in plastids (plastidial targeting) or in the cytosol (cytosolic targeting).

\section{Effect of EcHSK expression on amino acid levels} in source leaves

The effect of increased EcHSK gene expression on levels of aspartate-derived amino acids and of cys was tested. With respect to the commonly held assumption that, in higher plants, most of the de novo amino acid synthesis occurs in source leaves, this tissue was analysed for free amino acids using HPLC (Kreft et al., 2003). Analyses of amino acid levels revealed that in most of the transgenic plants, free levels of homoserine decreased
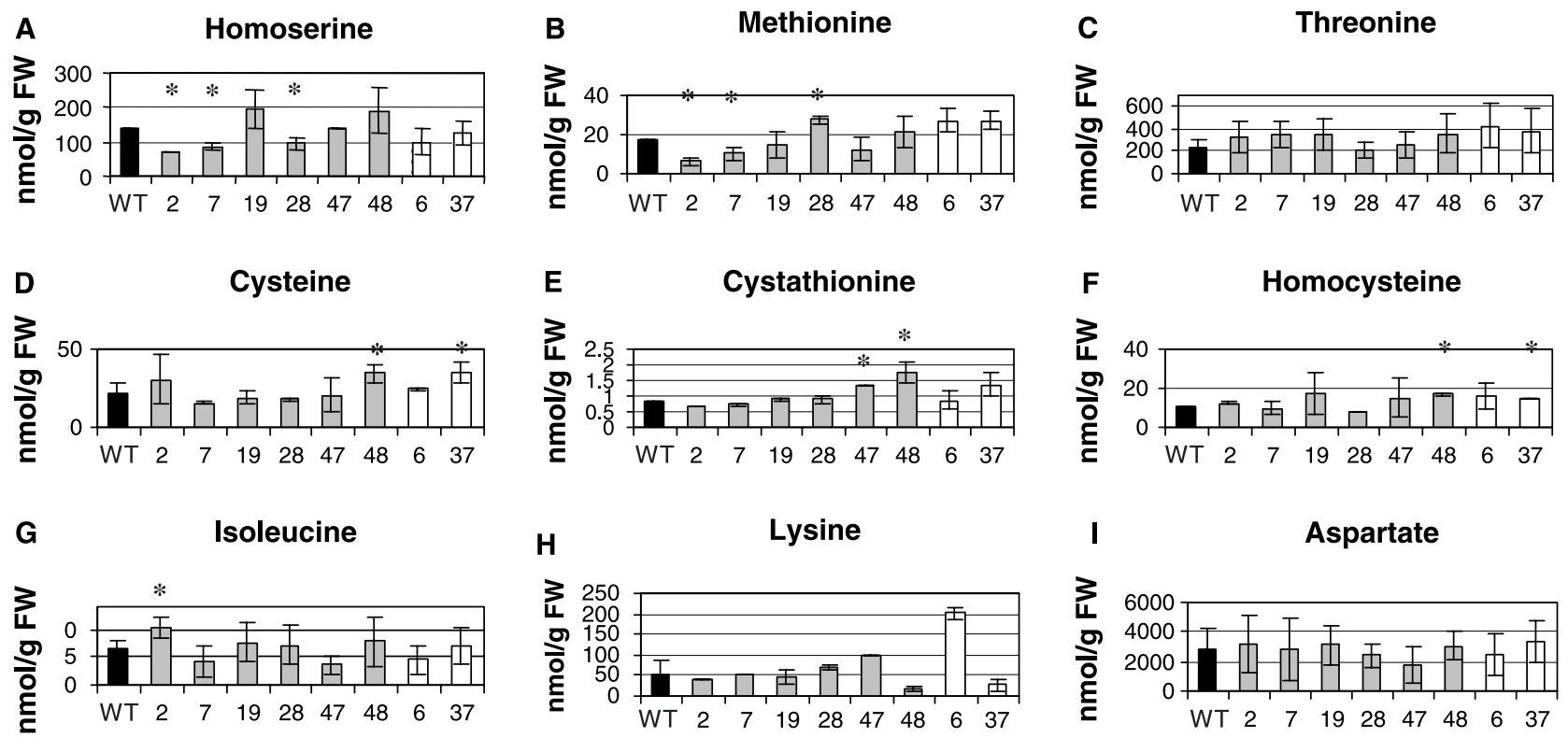

Fig. 2. Impact of increased HSK activity on the levels of selected amino acids. Metabolites were extracted from leaf tissues of 8-week-old plants (wild type: black bar; transgenic plants: grey bars for plastidial localisation and white bars for cytosolic localisation). A Homoserine, $\mathbf{B}$ methionine, $\mathbf{C}$ threonine, $\mathbf{D}$ cysteine, $\mathbf{E}$ cystathionine, $\mathbf{F}$ homocysteine, $\mathbf{G}$ isoleucine, $\mathbf{H}$ lysine, and $\mathbf{I}$ aspartate were determined by HPLC analysis with $O$-phtaldialdehyde derivatization and fluorescence detection. Amounts of amino acids are given in nanomoles per gram $\mathrm{FW}$ and represent mean values $\pm \mathrm{SD}(n=5)$. Asterisks indicate differences between wild type and transgenic plants analysed using Student's $t$-test that were statistically significant (asterisk: $P<0.05$ ) 
while met and thr contents increased, albeit inconsistently (Fig. 2A-C). In fact, met and thr levels were quite variable not only among lines, but within them. This is also the case for the other amino acids monitored by HPLC. However, the change in met is supported by the trend observed for the intermediates cystathionine (Fig. 2E) and homocysteine (Fig. 2F). Levels of ile (Fig. 2G), the amino acid downstream of thr, also increased tentatively.

It is important to note that a few lines showed slightly increased levels of lys (Fig. 2H) and nearly constant levels of asp (Fig. 2I). In some of the lines, cys increased (Fig. 2D) in a manner nearly identical to that observed for cystathionine and homocysteine. Nevertheless, as can be deduced by comparing Fig. 1A with Fig. 2, there was no clear correlation between total HSK activity and amino acid levels among the various transgenic plants, suggesting that met and thr metabolism are not regulated by HSK. Other metabolites determined by metabolite profiling via GC-MS analysis according to Nikiforova et al. (2005) were not affected by EcHSK overexpression (data not shown) but support the results of the amino acid measurements by HPLC. OPHS itself could not be determined due to its instability.

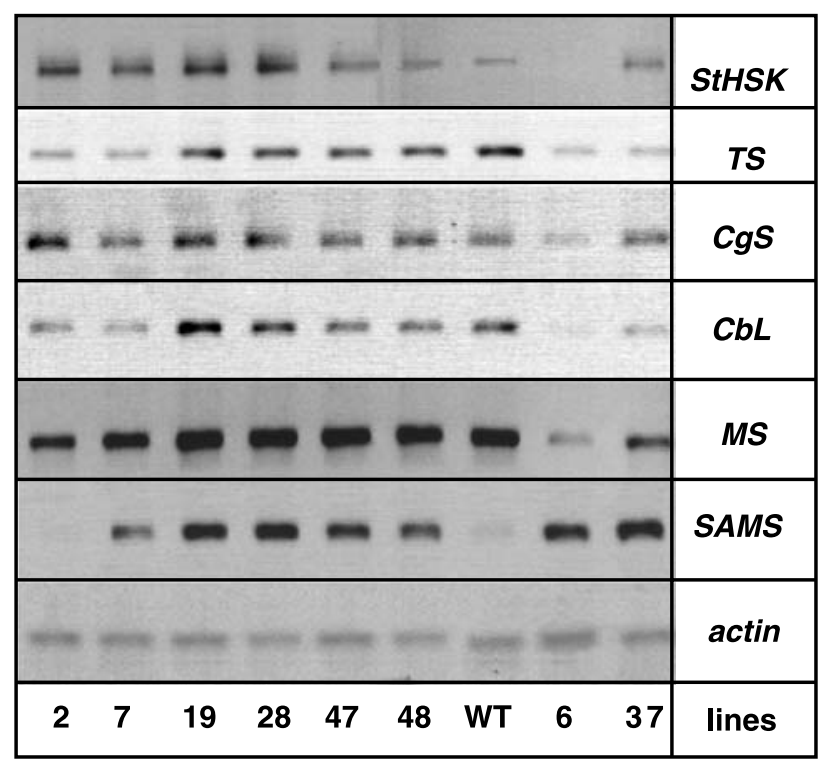

Fig. 3. Expression analysis of $H S K$ over-expressing plants and wild type (WT) by semi-quantitative RT-PCR. Total leaf RNA was extracted and $100 \mu \mathrm{g}$ of RNA was used to synthesise cDNA. Equality of amounts was tested with actin primer. Pathway-relevant genes such as endogenous HSK (StHSK), TS, CgS, CbL, MS and SAMS were tested by specific primer combinations of the potato genes. The nucleotide sequence of StHSK was submitted to the EMBL Data Library and assigned with the accession number AJ890444
Gene expression of aspartate pathway related enzymes was affected in plants over-expressing EcHSK

Total RNA was isolated from leaves of wild type control plants and transgenic lines over-expressing EcHSK in order to analyse gene expression of pathway related enzymes (Fig. 3). Semi-quantitative PCR analysis revealed that EcHSK over-expression did not influence the expression of most of the tested genes, i.e. the endogenous StHSK, TS, CgS, CbL and MS when targeted to chloroplasts. However, SAMS expression was stimulated in all transgenic plants except in line 2. In the transgenic plants with cytosolically localised EcHSK, the expression of other pathway genes was down regulated, except for $S A M S$, suggesting that cytosolically available OPHS might regulate the expression of pathway genes.

Feeding homoserine to detached petioles leads to a distinct increase in met and thr formation in leaves of both wild type and transgenic plants

When the results of the above experiments are taken together, it becomes clear that enhanced HSK activity in transgenic potato plants does affect levels of aspartate-derived metabolites, but that increases were less than expected from earlier experiments (Bartlem et al., 2000; Kim and Leustek, 2000; Zeh et al., 2001; Gakière et al., 2002; Lee et al., 2005). To look at this more closely, the effect of enhanced homoserine content on met and thr formation was analysed in leaves of wild type and transgenic plants. To this end, a feeding experiment with detached potato petioles of wild type plants and transgenic lines was performed. Freshly cut petioles were incubated either in MES buffer containing EDTA or in buffer plus homoserine at two different concentrations $(0.1$ and $1 \mathrm{mM})$. After $24 \mathrm{~h}$ in continuous light, metabolites were extracted and determined by HPLC analysis. As shown in Fig. 4, exogenously applied homoserine clearly increased met levels in leaves of both wild type and transgenic lines, but also increased thr levels. In most cases, met increased to an estimated threshold of about $30 \mathrm{nmol} / \mathrm{g}$ FW when plants were fed with $0.1 \mathrm{mM}$ homoserine. If this threshold was surpassed, e.g. by feeding $1 \mathrm{mM}$ homoserine, the internal met content dropped. However, the transgenic lines with exceedingly high levels of EcHSK expression were less affected.

Thr levels respond differently to homoserine feeding. Both applied concentrations of homoserine were able to increase internal thr levels but the highest levels were 
Line 7

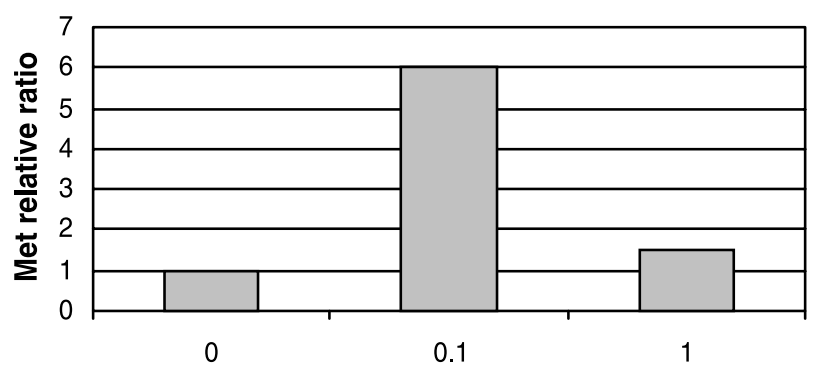

Line 47

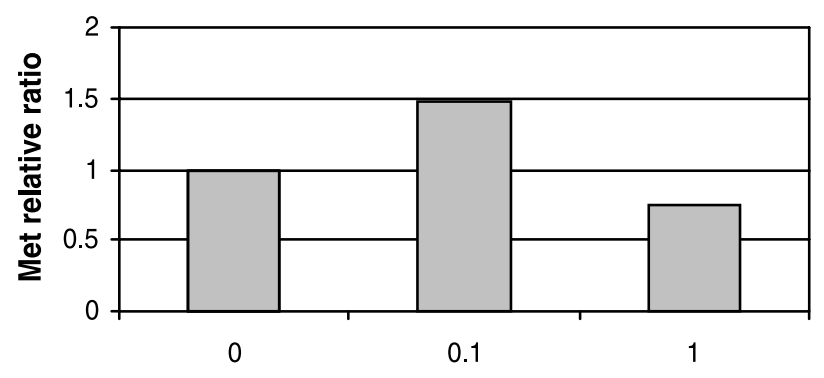

Line 37
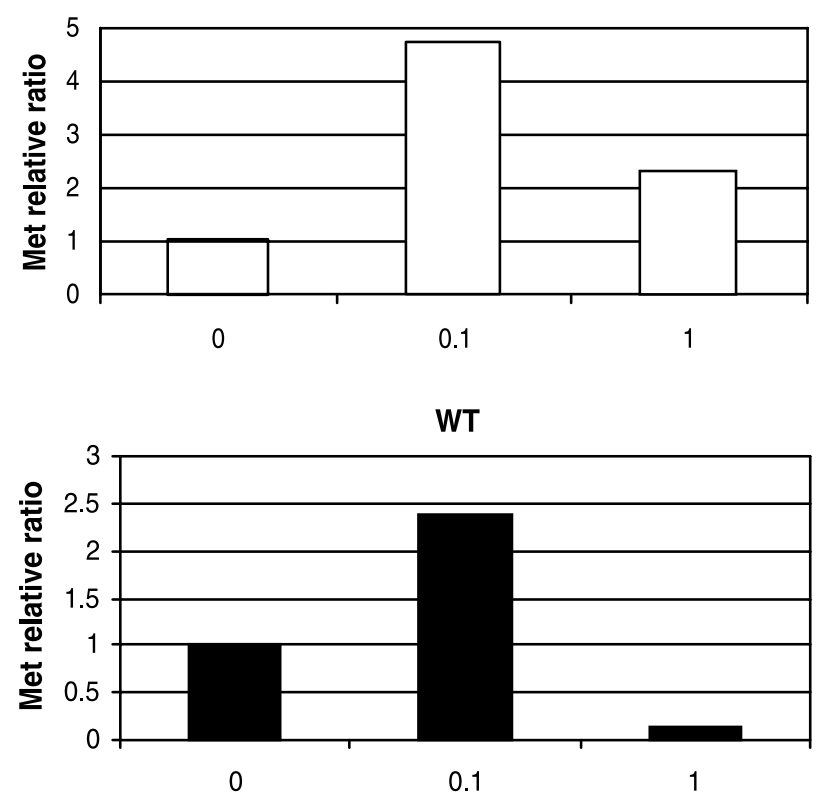

Line 7

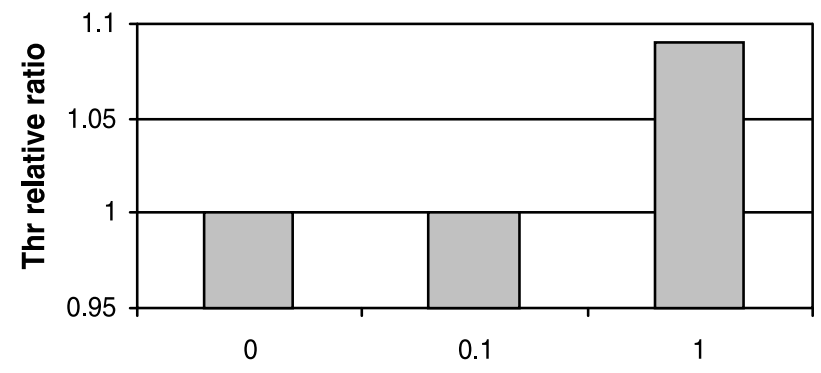

Line 47

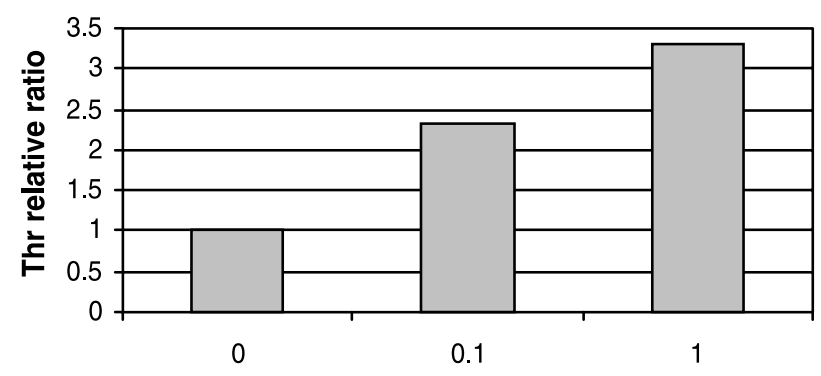

Line 37

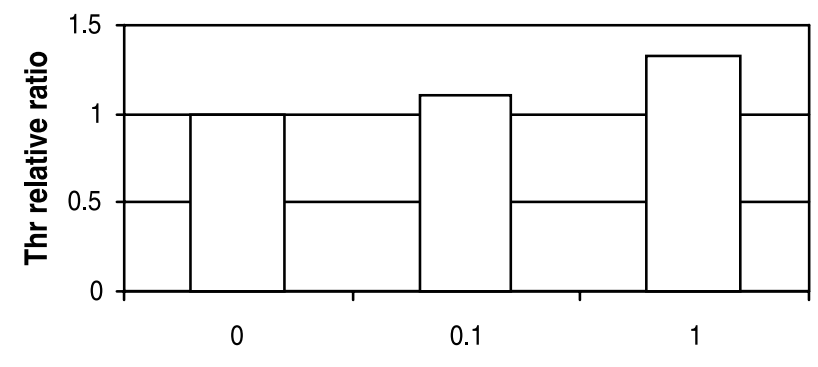

WT

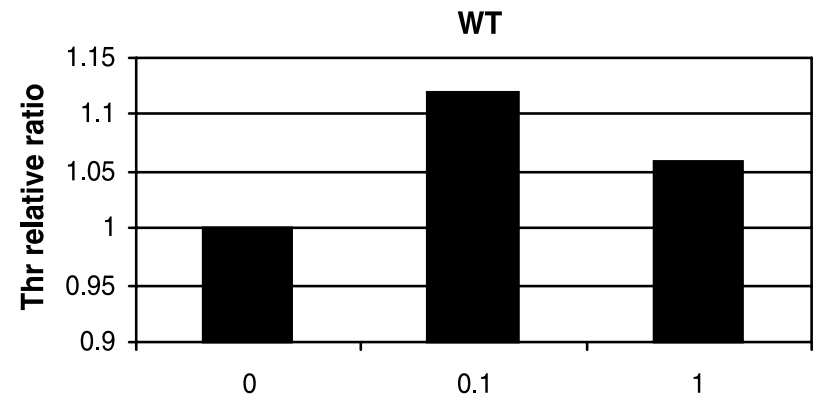

Fig. 4. Effect of supplying homoserine to detached petioles of potato wild type plants (WT, black bar) and transgenic plants (plastidial localisation: grey bars, Lines 7, 47; cytosolic localisation: white bar, line 37) expressing EcHSK on the Met and Thr formation. Petioles were freshly cut from 8-week old greenhouse grown plants, incubated either in $10 \mathrm{mM}$ MES-buffer containing $1 \mathrm{mM}$ EDTA or in buffer plus 0.1 and $1 \mathrm{mM}$ homoserine, respectively. Leaf material was sampled after $24 \mathrm{~h}$ of incubation and analysed by HPLC. The data are presented as the mean of a pool of five petiols of five individual plants per line, one measurement per line. Results are given as relative ratio normalised to the buffer control

achieved when met synthesis decreased. This suggests that even smaller amounts of homoserine are able to foster thr synthesis but that initially the majority of OPHS feeds into met synthesis.

\section{Discussion}

Met and thr are essential amino acids. Met serves as the precursor of SAM, which is likely the key metabolite in 
aspartate-derived amino acid metabolism (Hesse et al., 2004). Complex metabolic interactions within the network require tight regulation of synthesis and cellular homeostasis. The synthesis of aspartate family amino acids is regulated at several junctures (Hesse et al., 2004). Recent efforts have identified a couple of enzymes with the potential to act as suitable regulators (Galili et al., 2005). Several lines of evidence show that the branch point between $\mathrm{CgS}$ and TS plays a major regulatory role in the flux of carbon into met. Only when met/SAM pools are filled is TS activated to the degree that it can compete sufficiently with $\mathrm{CgS}$ for the common substrate OPHS (Ravanel et al., 1998; Hesse and Hoefgen, 2003; Hesse et al., 2004).

Here, total HSK activity was increased to elevate the flux of carbon towards synthesis of met and thr, the goal being to investigate the role of HSK in controlling the synthesis and homeostasis of met and thr. The results show that the synthesis of these amino acids is driven by the provision of the carbon backbone deriving from aspartate via homoserine. We drew this conclusion upon observing a set of transgenic plants in which total EcHSK transcript levels were elevated specifically in the cytosol or the chloroplast. At maximum, an 11-fold increase in enzymatic activity was observed with an average increase of 5-fold. However, although EcHSK was expressed by the $35 \mathrm{~S}$ promoter, the accumulation of transcript did not always correlate with increased enzymatic activity. Line 7 for example expressed low amounts of EcHSK RNA but the enzymatic activity increased to the same levels as did line 48 or 6 . Similar results have been observed when $\mathrm{CgS}, \mathrm{CbL}$ and $T S$ were expressed in potato (Maimann et al., 2001; Nikiforova et al., 2002; Kreft et al., 2003; respectively).

Earlier studies revealed that aspartate kinase regulates the overall flux of the carbon backbones for the synthesis of aspartate-derived amino acids such as lys and thr but not met (Galili, 1995). Taking this into consideration one would expect that met/thr levels would be enhanced if the equilibrium were shifted from homoserine to OPHS by phosphorylation. This is clearly the case here, since levels of homoserine decreased independent of the compartment to which EcHSK was targeted. A few of the transgenic plants display higher met and thr levels (double) than wild type plants, independent of subcellular EcHSK location. Intermediates such as cystathionine and homocysteine or the end-product ile tended to increase approximately 2 -fold. Other transgenic plants exhibited even lower met levels, which might be explained by a rapid rate of conversion to SAM or other products.
In a study by Lee et al. (2005) in the plant model Arabidopsis thaliana, over-expression of endogenous HSK had no effect on soluble OPHS, met, or thr levels; however, when treated with homoserine these plants produced far more OPHS than wild type and subsequently more met and thr. Here the over-expression of orthologous E. coli HSK in potato reveals that met and thr synthesis is driven by the availability of carbon backbone. These results and results obtained in earlier studies investigating the pathway in potato suggest that the regulation of met and thr metabolism is highly variable and is subject to complex biochemical, physiological, and environmental controls (Hesse and Hoefgen, 2003; Hesse et al., 2004). This is in agreement with the observation that the simple overexpression of HSK does not enable plants to overcome a certain threshold for met in the way that it does in transgenic plants over-expressing $\mathrm{CgS}$ or under-expressing TS (Muhitch, 1997; Bartlem et al., 2000; Kim and Leustek, 2000; Zeh et al., 2001; Gakière et al., 2002). In those studies the $\mathrm{CgS} / \mathrm{TS}$ ratio changed, resulting in higher met and thr levels, indicating the direction of pool filling: first met and then thr. Even significant increases in total HSK activity, independent of subcellular location, do not result in high met accumulation. Moreover, levels of the metabolites asp and lys are only weakly influenced by the HSK manipulation.

Changes in contents can be interpreted as trends; however, the intrinsic biological variation of metabolites in plants cannot be disregarded (Fiehn et al., 2000; Maimann et al., 2001; Riemenschneider et al., 2005). When EcHSK activity is high in transgenic plants, precursor (asp or homoserine) levels fluctuate since they are converted readily to downstream products. The marked tendency towards lys accumulation seen here ties in with the observation that a positive correlation exists between free lys and met in Arabidopsis modified in lys (Zhu and Galili, 2004) and in potato modified in cys metabolism (Nikiforova et al., 2002; Riemenschneider et al., 2005).

It is important to note that cys levels increased in lines with elevated cystathionine and homocysteine levels. Such an increase has also been noted in transgenic potato plants with down-regulated CbL activity (Maimann et al., 2000). In these plants, cystathionine accumulated in significant amounts. It may be that the increased cys levels seen here result from cystathionine cleaved enzymatically or by acid hydrolysis, resulting in elevated cys or homocysteine depending on which carbon backbone sulphide remains. However, this possibility remains unproven. Other nonpathway-related amino acids or metabolites did not show significant alterations in leaves (GC-MS data, not shown). 


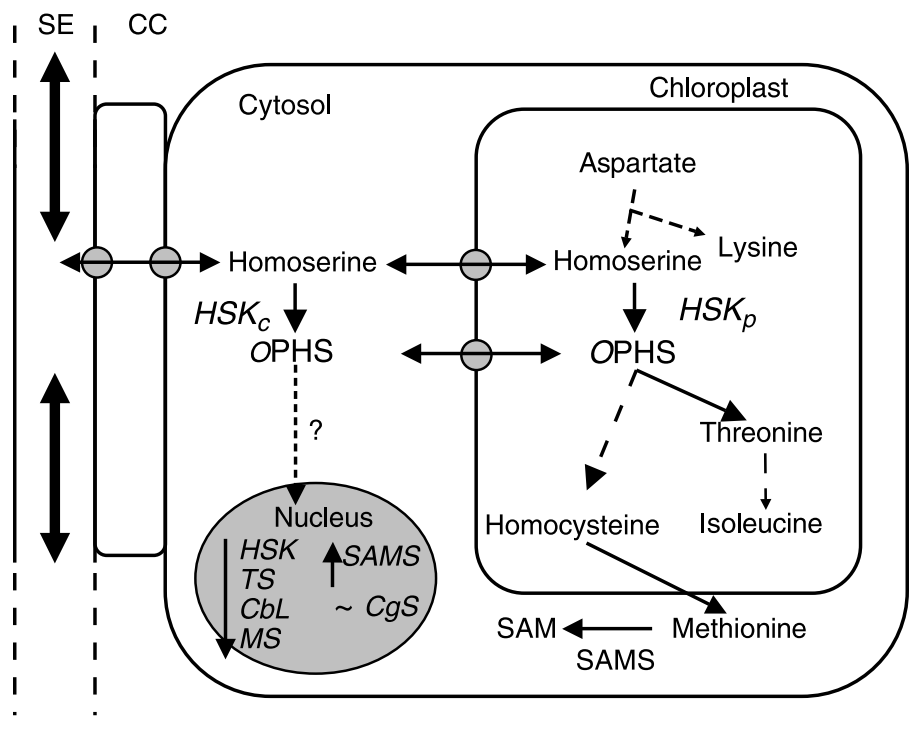

Fig. 5. Model for a signal transduction regulating pathway relevant gene expression by cytosolically localised $O$-phosphohomoserine (OPHS). HSK Homoserine kinase, TS thr synthase, $C g S$ cystathionine $\gamma$-synthase, $C b L$ cystathionine $\beta$-lyase, $H S K_{p}$ plastidial targeted homoserine kinase, $H S K_{c}$ cytosolically targeted homoserine kinase, $M S$ met synthase, $\operatorname{SAM}(S) S$-adenosylmethionine (synthetase); $S E$ sieve elements; $C C$ companion cell. Arrows indicate up or down regulation of gene expression, $\sim$ indicate nearly constant expression
Thus, the data support a model in potato in which the met pool is filled prior to the thr pool (Hesse and Hoefgen, 2003; Hesse et al., 2004; Fig. 5).

Although the analysis revealed a significant increase in total HSK activity flow towards met, met synthesis was not enhanced, indicating that endogenous StHSK levels do not limit the synthesis of met and thr. Rather the amount of aspartate-derived carbon seems to check these downstream metabolites. More support for this idea comes from the observation that met and thr levels rise when homoserine is supplied to detached potato leaves via the petiole. Because exogenously supplied homoserine had a stimulating effect on met and thr biosynthesis in both wild type and transgenic plants, it appears that in wild type plants the in vivo concentration of homoserine is the limiting factor for HSK activity. In this context, it is important to note that in wild type plants, high homoserine concentrations reduced the thr level. It is known that thr down-regulates its own synthesis by feedback inhibition of homoserine dehydrogenase (Galili, 1995); this regulatory mechanism was overridden here since the reaction product, homoserine, was fed in excess. Thus, it might be possible that in wild type plants, HSK activity is limiting but that also homoserine levels need tight control. This seems not to be the case in transgenic plants.

These results in potato corroborate the recent findings of Lee et al. (2005) in Arabidopsis. Transgenic Arabidopsis plants over-expressing endogenous $H S K$ exhibited increased met and thr levels when they were fed with homoserine. This effect was more pronounced when transgenic plants over-expressing endogenous $\mathrm{AtCgS}$ and or- thologous EcTS were fed with homoserine. These authors concluded that homoserine supply is the major factor limiting the synthesis of OPHS, met, and thr.

In this study, HSK activity does not seem to regulate the expression of pathway relevant genes, at least in plants expressing chloroplast-targeted EcHSK, which is supported by the fact that there are no changes in expression of StHSK, CgS, CbL, MS, and TS. However, in plants expressing HSK cytosolically, the expression of these very genes was reduced, indicating a possible role for OPHS in regulating the expression of pathway-relevant genes. An exception is SAMS, which increased in expression regardless of where EcHSK was localised. Only in line 2 was such an increase not observed. Since met levels increased, it may be that met was subsequently converted to SAM (undetermined). The increase of SAMS expression indirectly indicates an increase of flux from met to SAM, which would in turn exert its known regulatory function (Fig. 5).

The increase in met and thr levels in plants with EcHSK targeted to both the chloroplast and cytosol indicate that OPHS is exchanged between compartments, at least between these compartments (Fig. 5). Even when EcHSK is expressed in a 'foreign' compartment, the cytosol, where the rest of the pathway is absent, met and thr levels were increased. Thus, both homoserine and OPHS are able to cross the chloroplast membrane by carriers as suggested by Aubert et al. (1998). In this context, it is of interest why homoserine/OPHS is exported from the chloroplast and how homeostasis is kept and sensed. Expression analysis revealed in this study that cytosolically localised OPHS regulates gene expression of 
pathway genes (Figs. 3 and 5). One can assume that the export of homoserine/OPHS is necessary to deliver precursors for amino acid synthesis to other organs such as sink organs or that it negatively regulates the de novo synthesis of its end products to keep met and thr in balance (Fig. 5).

The export of homoserine seems like a reasonably likely phenomenon since phloem sap contains significant amounts of homoserine, which is, at least in legumes, transported to developing seeds only able to synthesise met and thr autotrophically in later phases of their development (Rochat and Boutin, 1991). Furthermore, there is evidence that pathogens specialise to use homoserine as a host signal to trigger the expression of pathogenesis genes (Yang et al., 2005). Also, derivatives of homoserine such as homoserine lactone serve as signal substances involved in microbiological quorum sensing, enabling bacteria to communicate and coordinate behaviour and responses to nutritional status via signalling molecules (Bauer and Mathesius, 2004). Because of their reduced levels of homoserine, it would be interesting to investigate the transgenic HSK potato plants to see if they are more resistant to pathogen infection, as they show reduced homoserine content.

These results in potato, a crop plant, corroborate and extend work done in Arabidopsis, a model plant, and flesh out new ideas of how aspartate-derived amino acids are regulated. Further, a new model of gene regulation and consequently of aspartate-derived amino acid homeostasis can be drawn based on the data presented here (Fig. 5). In this model, homoserine/OPHS levels drive gene expression and act as indicators of nutritional status within the plant system.

\section{Acknowledgments}

We wish to thank Romy Ackermann for performing the potato transformations, the gardeners for excellent greenhouse work, and Josef Bergstein for photographical assistance. We are grateful to Megan McKenzie for her engaged editing of the manuscript (www.sciencewriting.net). The work was supported financially by the European Union (QLRT-2000-00103).

\section{References}

Aubert S, Curien G, Bligny R, Gout E, Douce R (1998) Transport, compartmentation, and metabolism of homoserine in higher plant cells. Plant Physiol 116: 547-557

Bartlem D, Lambein I, Okamoto T, Itaya A, Uda Y, Kijima F, Tamaki Y, Nambara E, Naito S (2000) Mutation in the threonine synthase gene results in an over-accumulation of soluble Met in Arabidopsis. Plant Physiol 123: 101-110

Bauer WD, Mathesius U (2004) Plant responses to bacterial quorum sensing signals. Curr Opin Plant Biol 7: 429-433
Baum HJ, Madison JT, Thompson JF (1983) Feedback inhibition of homoserine kinase from radish leaves. Phytochem 22: 2409-2412

Chiba Y, Ishikawa M, Kijima F, Tyson RH, Kim J, Yamamoto A, Mambara E, Leustek T, Wallsgrove RM, Naito S (1999) Evidence for autoregulation of cystathionine $\gamma$-synthase mRNA stability in Arabidopsis. Science 286: 1371-1374

Curien G, Dumas R, Ravanel S, Douce R (1996) Characterization of an Arabidopsis thaliana cDNA encoding an S-adenosylmethionine-sensitive threonine synthase. FEBS Lett 390: 85-90

Curien G, Job D, Douce R, Dumas R (1998) Allosteric activation of Arabidopsis threonine synthase by S-adenosylmethionine. Biochem 31 : 13212-13221

Deblaere R, Bytebier B, de Greve H, Debroek F, Schell J, van Montagu M, Leemanns J (1985) Efficient octopine Ti plasmid-derived vectors of Agrobacterium mediated gene transfer to plants. Nucleic Acids Res 13: 4777-4788

Fiehn O, Kopka J, Dörmann P, Altmann T, Trethewey RN, Willmitzer L (2000) Metabolite profiling for plant functional genomics. Nat Biotech 18: $1157-1161$

Gakière B, Denis L, Droux M, Job D (2002) Over-expression of cystathionine $\gamma$-synthase in Arabidopsis thaliana leads to increased levels of methionine and S-methylmethionine. Plant Physiol Biochem 40: $119-126$

Galili G (1995) Regulation of lysine and threonine synthesis. Plant Cell 7: 899-906

Galili G, Amir R, Hoefgen R, Hesse H (2005) Improving the levels of essential amino acids and sulfur metabolites in plants. Biol Chem 386 : $817-831$

Greenberg JM, Thompson JF, Madison JT (1988) Homoserine kinase and threonine synthase in methionine-overproducing soybean tissue cultures. Plant Cell Rep 7: 477-480

Harms K, von Ballmoos P, Brunold C, Höfgen R, Hesse H (2000) Expression of a bacterial serine acetyltransferase in transgenic potato plants leads to increased levels of cysteine and glutathione. Plant J 22: 335-343

Hesse H, Hoefgen R (2003) Molecular aspects of methionine biosynthesis in Arabidopsis and potato. Trends Plant Sci 8: 259-262

Hesse H, Kreft O, Maimann S, Zeh M, Hoefgen R (2004) Current understanding of the regulation of methionine biosynthesis in plants. J Exp Bot 55: 1799-1808

Höfgen R, Willmitzer L (1990) Biochemical and genetic analysis of different patatin isoforms expressed in various organs of potato (Solanum tuberosum). Plant Sci 66: 221-230

Kim J, Leustek T (2000) Repression of cystathionine $\gamma$-synthase in Arabidopsis thaliana produces partial methionine auxotrophy and developmental abnormalities. Plant Sci 151: 9-18

Kreft O, Höfgen R, Hesse H (2003) Functional analysis of cystathionine $\gamma$ synthase in genetically engineered potato plants. Plant Physiol 131: 1843-1854

Lee M, Leustek T (1999) Identification of the gene encoding homoserine kinase from Arabidopsis thaliana and characterization of the recombinant enzyme derived from the gene. Arch Biochem Biophys 372: 135-142

Lee M, Martin MN, Hudson AO, Muhitch MJ, Leustek T (2005) Methionine and threonine synthesis are limited by homoserine availability and not the activity of HSK in A. thaliana. Plant J 41: 685-696

Lindroth P, Mopper K (1979) High performance liquid chromatographic determination of subpicomole amounts of amino acids by precolumn fluorescence derivatization with $o$-phthaldialdehyde. Anal Chem 51: 1667-1674

Logemann J, Schell J, Willmitzer L (1987) Improved method for the isolation of RNA from plant tissues. Anal Biochem 163: 16-20

Maimann S, Wagner C, Kreft O, Zeh M, Willmitzer L, Höfgen R, Hesse $\mathrm{H}$ (2000) Transgenic potato plants reveal the indispensable role of 
cystathionine beta-lyase in plant growth and development. Plant $\mathrm{J} 23$ : 747-758

Maimann S, Höfgen R, Hesse H (2001) Enhanced cystathionine $\beta$-lyase activity in transgenic potato plants does not force metabolite flow towards methionine. Planta 23: 163-170

Muhitch MJ (1997) Effects of expressing E. coli threonine synthase in tobacco (Nicotiana tabacum) suspension culture cells on free amino acid levels, aspartate pathway enzyme activities and uptake of aspartate into the cells. J Plant Physiol 150: 16-22

Muhitch MJ, Wilson KG (1983) Chloroplasts are the subcellular localisation of both soluble and membrane-associated homoserine kinase in pea (Pisum sativum L.) leaves. Z Pflanzenphysiol 110: 39-46

Murashige T, Skoog F (1962) A revised medium for rapid growth and bioassays with tobacco tissue cultures. Physiol Plant 15: 473-497

Nikiforova V, Kempa S, Zeh M, Maimann S, Kreft O, Casazza AP, Riedel K, Tauberger E, Höfgen R, Hesse H (2002) Engineering of cysteine and methionine biosynthesis in potato. Amino Acids 22: 259-278

Nikiforova VJ, Kopka J, Tolstikov V, Fiehn O, Hopkins L, Hawkesford MJ, Hesse H, Hoefgen R (2005) Systems rebalancing of metabolism in response to sulfur deprivation, as revealed by metabolome analysis of Arabidopsis plants. Plant Physiol 138: 304-318

Ravanel S, Gakiere B, Job D, Douce R (1998) The specific features of methionine biosynthesis and metabolism in plants. Proc Natl Acad Sci USA 95: 7805-7812

Riemenschneider A, Riedel K, Hoefgen R, Papenbrock J, Hesse H (2005) Impact of reduced $O$-acetylserine(thiol)lyase isoform contents on potato (Solanum tuberosum L.) plant metabolism. Plant Physiol 137: 892-900

Riesmeier J, Klonus D, Pohlenz HD (1993) Purification to homogenity and characterisation of homoserin kinase from wheat germ. Phytochemistry 32 : $581-584$
Rocha-Sosa M, Sonnewald U, Frommer W, Stratmann M, Schell J, Willmitzer L (1989) Both developmental and metabolic signals activate the promoter of the class I patatin gene. EMBO J 8: 23-29

Rochat C, Boutin JP (1991) Metabolism of phloem-borne amino acids in maternal tissues of fruit of nodulated or nitrate-fed pea plants. J Exp Bot 42: $207-221$

Sambrook J, Fritsch EF, Maniatis T (1989) Molecular cloning: a laboratory manual, 2nd ed. Cold Spring Harbor Laboratory Press, Cold Spring Harbor

Thoen A, Rognes SE, Aarnes H (1978) Biosynthesis of threonine from homoserine in pea-seedlings. 2. Threonine synthase. Plant Sci Lett 13: 113-119

Wallsgrove RM, Lea PJ, Miflin BJ (1983) Intracellular localisation of aspartate kinase and the enzymes of threonine and methionine biosynthesis in green leaves. Plant Physiol 71: 780-784

Yang Z, Rogers LM, Song Y, Guo W, Kolattukudy PE (2005) Homoserine and asparagine are host signals that trigger in planta expression of a pathogenesis gene in Nectria haematococca. Proc Natl Acad Sci USA 102: 4197-4202

Zeh M, Casazza AP, Kreft O, Rössner U, Biebrich K, Willmitzer L, Höfgen R, Hesse H (2001) Antisense inhibition of threonine synthase leads to high methionine content in transgenic potato plants. Plant Physiol 127: 792-802

Zhu X, Galili G (2004) Lysine metabolism is concurrently regulated by synthesis and catabolism in both reproductive and vegetative tissues. Plant Physiol 135: 129-136

Authors' address: Dr. Holger Hesse, Department of Molecular Physiology, Max-Planck-Institut für Molekulare Pflanzenphysiologie, Am Mühlenberg 1, D-14476 Golm, Germany,

Fax: +49 33156789 8247, E-mail: hesse@mpimp-golm.mpg.de 